\title{
Measles virus infection diminishes preexisting antibodies that offer protection from other pathogens
}

\section{Mina, Michael J.}

2019-11-01

Mina, M J , Kula , T , Leng , Y, Li , M , de Vries , R D , Knip , M , Siljander , H , Rewers , M , Choy, D F , Wilson, M S , Larman, H B , Nelson , A N, Griffin, D E , de Swart , R L \& Elledge , S J 2019 , ' Measles virus infection diminishes preexisting antibodies that offer protection from other pathogens ' , Science , vol. 366 , no. 6465 , pp. 599-+ . https://doi.org/10.1126/science.aay648!

http://hdl.handle.net/10138/307628

https://doi.org/10.1126/science.aay6485

publishedVersion

Downloaded from Helda, University of Helsinki institutional repository.

This is an electronic reprint of the original article.

This reprint may differ from the original in pagination and typographic detail.

Please cite the original version. 
VIRAL IMMUNOLOGY

\section{Measles virus infection diminishes preexisting antibodies that offer protection from other pathogens}

\author{
Michael J. Mina ${ }^{1,2,3 *} \dagger$, Tomasz Kula, ${ }^{1,2}$, Yumei Leng ${ }^{1}$, Mamie Li ${ }^{2}$, Rory D. de Vries ${ }^{4}$, Mikael Knip ${ }^{5,6}$, \\ Heli Siljander ${ }^{5,6}$, Marian Rewers ${ }^{7}$, David F. Choy ${ }^{8}$, Mark S. Wilson ${ }^{8}$, H. Benjamin Larman ${ }^{9}$, \\ Ashley N. Nelson ${ }^{10} \neq$, Diane E. Griffin ${ }^{10}$, Rik L. de Swart ${ }^{4}$, Stephen J. Elledge ${ }^{1,2,11}$
}

\begin{abstract}
Measles virus is directly responsible for more than 100,000 deaths yearly. Epidemiological studies have associated measles with increased morbidity and mortality for years after infection, but the reasons why are poorly understood. Measles virus infects immune cells, causing acute immune suppression. To identify and quantify long-term effects of measles on the immune system, we used VirScan, an assay that tracks antibodies to thousands of pathogen epitopes in blood. We studied 77 unvaccinated children before and 2 months after natural measles virus infection. Measles caused elimination of 11 to $73 \%$ of the antibody repertoire across individuals. Recovery of antibodies was detected after natural reexposure to pathogens. Notably, these immune system effects were not observed in infants vaccinated against MMR (measles, mumps, and rubella), but were confirmed in measles-infected macaques. The reduction in humoral immune memory after measles infection generates potential vulnerability to future infections, underscoring the need for widespread vaccination.
\end{abstract}

| the time before vaccination, nearly every child experienced measles, which resulted in millions of deaths. Global measles vaccination efforts have led to logarithmic reductions in the incidence of measles virus (MV) infections and measles-related mortality. However, measles remains endemic in much of the world, affecting $>7$ million people annually and causing $>100,000$ deaths (1-3). After decades of decline, the number of worldwide cases of measles has increased by nearly $300 \%$ since 2018 as a result of reduced vaccination (2). This increase is likely to be accompanied by substantial mortality risks (3). The resurgence of measles underscores the importance of understanding the full consequences of MV infection and accurately estimating the value of measles vaccination (4).

Immunosuppression was first documented when children with measles showed negative cutaneous tuberculin reactions after previously testing positive (5). Subsequent studies have shown decreased interferon signaling, skewed cytokine responses, lymphopenia, and suppression of lymphocyte proliferation shortly after infection (6). The MV receptor CD150/ SLAMF1 (signaling lymphocytic activation molecule family member 1) is highly expressed on memory $\mathrm{T}, \mathrm{B}$, and plasma cells, resulting in their infection and depletion without an effect on total immunoglobulin $\mathrm{G}$ (IgG) levels (7-12). Recovery of the functional immune response, including resolution of lymphopenia, occurs 2 to 4 weeks after viral clearance $(6,10,13,14)$. However, MV replication in immune cells has been hypothesized to impair immune memory, potentially causing "immunological amnesia" $(10,15,16)$.

Most bona fide immune memory cells reside in the lymphoid tissues and bone marrow (17-20). Peripheral blood mononuclear cells are often used for evaluating immunological memory repertoires. However, these cells are in relative flux owing to recent infections, which limits their utility for measuring long-term immune memory. Antibodies are thought to better represent long-lived humoral memory $(18,20)$. Most antibodies in the peripheral blood are produced by bone marrow long-lived plasma cells (LLPCs) and are impervious to disruptions in peripheral memory cells (17-22). Changes in pathogen-specific antibodies measured in the peripheral blood reflect changes in the long-lived permanent memory repertoire.

Epidemiological evidence has associated MV infections with increases in morbidity and mortality for as long as 5 years $(15,23)$ and suggests that in the pre-vaccine era, MV may have been associated with up to $50 \%$ of all childhood deaths from infectious diseases, mostly from non-MV infections (15). This phenomenon might be explained by immune amnesia. However, to date, no study has successfully resolved whether measles-induced immune amnesia-a reduction in the diversity of the immune memory repertoire after measles infections-indeed exists. To address this issue, we have studied paired blood samples collected before and after MV infection using a seroprofiling tool that allows the detection of thousands of pathogen-specific antibodies.

\section{Measuring the consequences of measles on immune memory}

During a recent measles outbreak in the Netherlands, families in communities with low vaccination rates consented to provide blood samples. Plasma was collected before and after laboratory-confirmed MV infection from 77 unimmunized children with a mean age of $9(\mathrm{SD} \pm 2)$ years, plus five unimmunized children who remained uninfected during the study (24). Of the 77 children, 34 were reported to have mild measles and 43 to have severe measles [detailed in (24)]. The mean time between sample collections was 10 weeks, and mean time of collection after MV infection was 7 weeks (table S1).

To measure the diversity and magnitude of the epitope-specific antibody repertoires in these children and controls, we used VirScan (25), a phage-display immunoprecipitation and sequencing (PhIP-Seq) technology (26) developed for virome-wide detection of antibodies against viral epitopes. VirScan primarily detects antibodies to short contiguous epitopes as opposed to conformational epitopes. The cells producing antibodies to all epitopes are phenotypically similar, aside from their antibody product. Thus, changes in the antibody repertoire detected by VirScan represent changes across the spectrum of antibodies, and these include neutralizing and non-neutralizing antibodies. For this study, we generated an expanded VirScan library that encodes the full proteomes of most known human pathogenic viruses ( 400 species and strains) plus many bacterial proteins. For each sample, we obtained a comprehensive measure of the individual's antipathogen antibody repertoire diversity (i.e., the total epitope hits across all pathogen peptides). We also derived an antibody epitope binding signal (EBS), which is

\footnotetext{
'Division of Genetics, Brigham and Women's Hospital, Howard Hughes Medical Institute, Boston, MA 02115, USA. ²Department of Genetics, Harvard Medical School, Boston, MA 02115, USA.

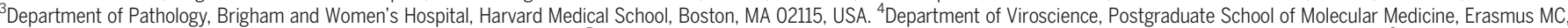

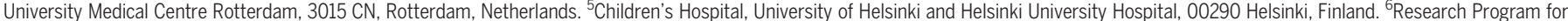

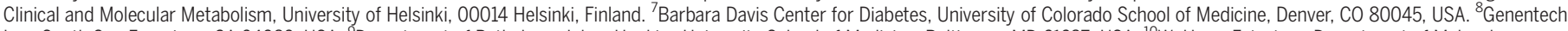
Inc., South San Francisco, CA 94080, USA. ${ }^{9}$ Department of Pathology, Johns Hopkins University School of Medicine, Baltimore, MD 21287, USA. ${ }^{10}$ W. Harry Feinstone Department of Molecular Microbiology and Immunology, Johns Hopkins Bloomberg School of Public Health, Baltimore, MD 21205, USA. ${ }^{11}$ Program in Virology, Harvard Medical School, Boston, MA 02115, USA.

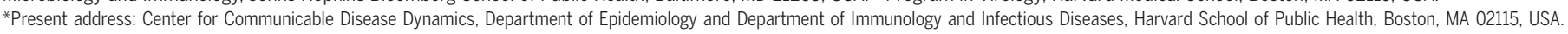

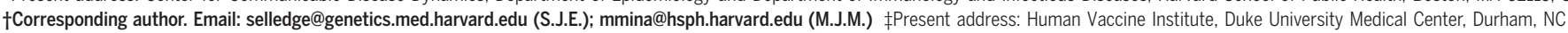
27710, USA.
} 
A

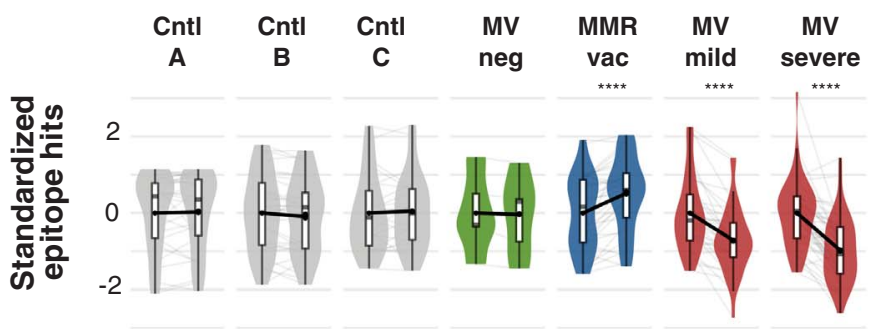

B

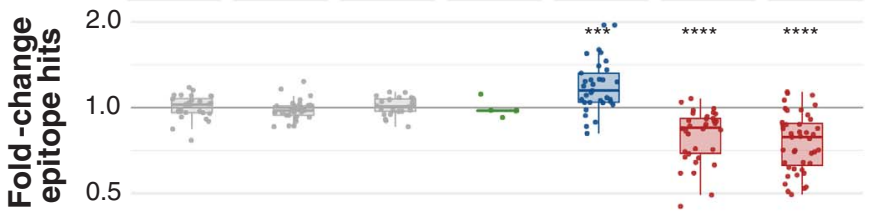

C

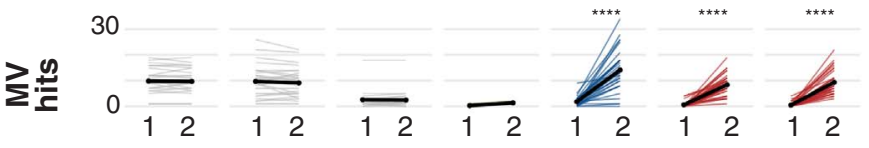

E

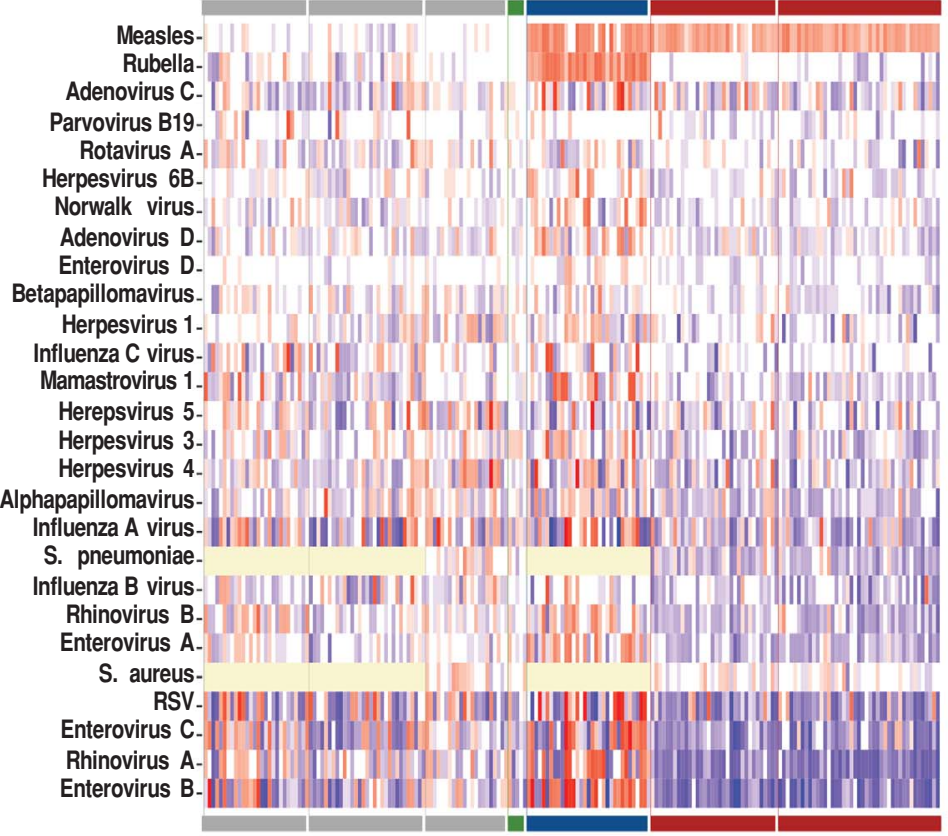

Change in Ab hits $\left(\log _{10}\right.$ scale)

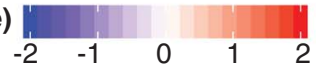

D Enterovirus

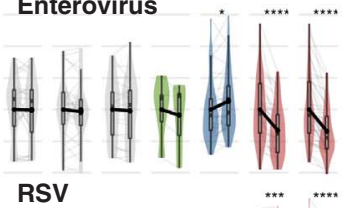

$H H H A H A$

H H H H

Influenza virus

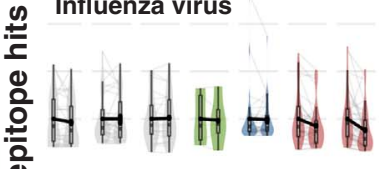

Coronavirus

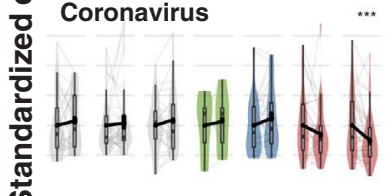

Herpesvirus

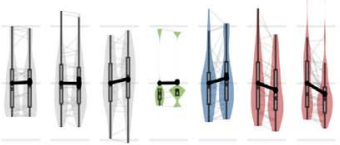

Papillomavirus

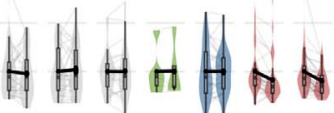

Adenovirus

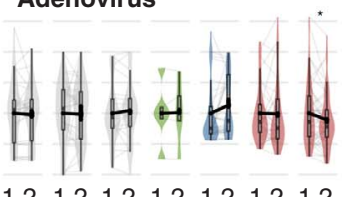

12121212121212 Time point
Fig. 1. Measles virus infections reduce antibody diversity.

(A) Total epitopes recognized at time 1, left, and time 2, right, per cohort. For comparison across cohorts, values are standardized across all samples per cohort to a mean of 0 and standard deviation of

1. Each gray line indicates a paired sample from an individual, and black connecting lines indicate mean change from zero. Boxplots indicate interquartile range and median. Asterisks indicate paired $t$ test $P$ values. (B) Fold change of total antibody diversity (i.e., number of total epitope hits) at time 2 versus time 1. Each point represents one paired sample from an individual. Boxplots indicate interquartile range and median. Asterisks indicate significant differences relative to control A (Cntl A), based on student's $t$ test $P$ values. (C) Number of measles epitope hits per sample at time 1 and time 2. Thin lines indicate paired samples. Black lines indicate cohort averages. (D) As in (A), but for individual viruses. Bonferronicorrected $P$ values in (A to D): ${ }^{*} P<$ 0.05 , ${ }^{* *} P<0.001$, ${ }^{* * *} P<0.001$, ${ }^{* * * *} P<0.0001$. (E) Heatmap indicating the change in the total number of epitope hits per species between time 1 and time 2. Each column represents an individual paired sample and each row a pathogen. Cohorts are indicated by the solid bars at top and bottom and are in the same order (left to right) as in (A). Yellow cells indicate that pathogen was not assayed in those samples. Ab, antibody. a relative measure of antibody titer for each epitope.

We used VirScan to profile the immune memory antibody repertoires before (time 1) and after (time 2) MV infection. Paired samples were also obtained from four control cohorts ( $n=119$ paired specimens; table $\mathrm{S} 1$ ). These samples were derived from: (i) approximately age-matched controls sampled at similar intervals ( $\sim 3$ months) as the measles cohorts (control A; $n=28$ paired specimens); (ii) age-matched controls with samples collected $\sim 1$ year apart (control $\mathrm{B} ; n=31$ ); (iii) adult controls with collection intervals similar to the measlesinfected individuals (control C; $n=22$ ); (iv) young children before and after their first measles-mumps-rubella (MMR) vaccination
(MMR vaccinated; $n=33$ ); and (v) unvaccinated children from the same community as the MV cases but who remained seronegative for MV (MV negative; $n=5$ ). Control cohorts A, $\mathrm{B}$, and $\mathrm{C}$ were individuals with no known exposure to MV.

\section{Measles modulates the diversity of the antibody repertoire and causes loss of preexisting antibodies}

We assessed changes in antibody repertoire diversity (measured as the total number of unique pathogen epitopes recognized, or epitope hits) before and after measles relative to those observed in controls, standardizing the total number of epitope hits per individual by cohort for comparison (Fig. 1A). We detected substantial reductions in the number of pathogen epitopes recognized after measles but limited changes in the absence of measles. MV infections were associated with a mean reduction of $\sim 20 \%$ in the overall diversity or size of the antibody repertoire measured by VirScan (Fig. 1B), and this was consistent across individual pathogens (Fig. 1, D and E, and fig. S1). However, effect sizes varied. Notably, 12 of the 77 children (16\%) lost $>40 \%$ of their overall antibody repertoire diversity. We detected increases in MV-specific epitopes in children after measles infection or MMR vaccination (Fig. 1C). No changes in the total IgG, IgA, or IgM levels were detected, as determined by quantitative ELISA (enzyme-linked immunosorbent assay) (fig. S2). These results suggest 
A

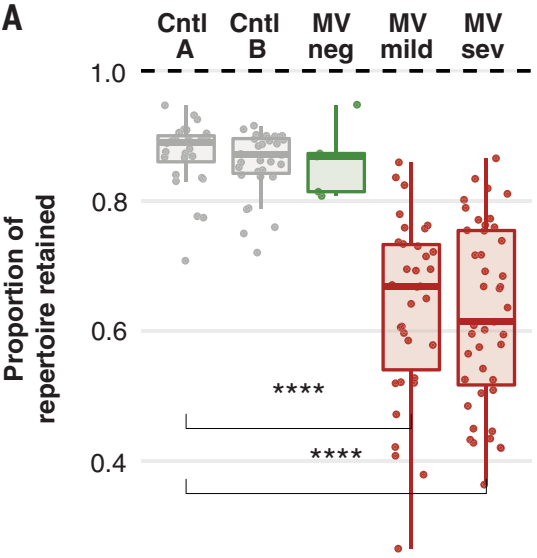

B

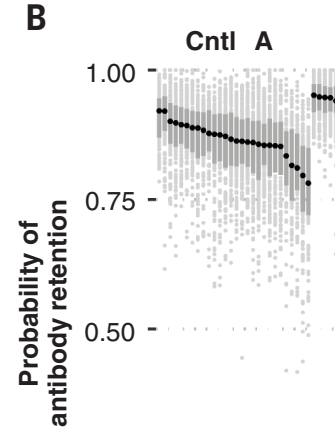

$0.25-$

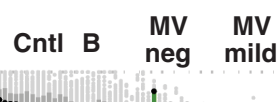

MV
mild

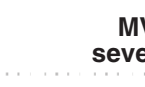

MV
severe

C

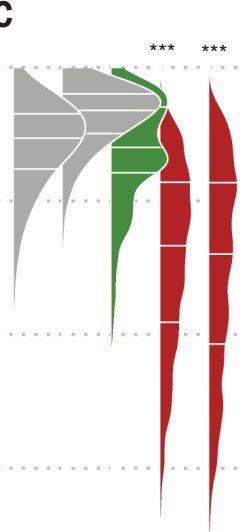

Fig. 2. Measles eliminates preexisting immune memory. (A) The proportion of total epitopes detected at time 1 that were retained at time 2. One point represents one child. Bonferroni-adjusted student's $t$ test $P$ values for significant differences relative to control $A$ are shown (**** $P<0.0001$ ). (B) Probability of retaining initial antibodies at time 2 for individual pathogens per child. Each point represents a single pathogen per child. Probabilities are obtained by fitting a binomial random effects model controlling for interval duration (materials and methods). Each boxplot represents the interquartile range and median retention probabilities calculated across pathogens for a single child. Points indicate pathogen-specific retentions that are outside of the interquartile range per child. Children are rank ordered along the $x$ axis by the median (black dot per boxplot) retention probabilities. (C) Density distribution of probabilities shown in (B), derived by collapsing all points in (B) by cohort. Bonferroni-adjusted Wilcoxon signed rank test $P$ values for differences relative to control $A$ are shown $\left({ }^{* * *} P<0.001\right)$. that, rather than a simple loss of total IgG, there is a restructuring of the antibody repertoire after measles.

To measure the full effect of measles on the pre-measles repertoire and to circumvent interference from new exposures during follow-up, we next restricted analysis to epitopes detected at the first time point and quantified retention or loss of epitope recognition. After severe or mild measles, children lost a median of $40 \%$ (range: 11 to $62 \%$ ) or $33 \%$ (range: 12 to $73 \%$ ), respectively, of their total preexisting pathogenspecific antibody repertoires (Fig. 2A and fig. S3). In contrast, controls retained $\sim 90 \%$ of their repertoires over similar or longer durations.

Controlling for interval duration in a binomial random effects model (see materials and methods), we estimated the per-pathogen probability of antibody retention for each child (Fig. 2, B and C). Loss of antibodies after MV infection varied widely for specific pathogens and between children. A small fraction of MVinfected individuals retained antibodies similar to the bottom quartile of the controls. However, the most-affected $20 \%$ of children lost $>50 \%$ of the pathogen-specific antibodies for most pathogens. In some of these children, up to $70 \%$ loss was detected for specific pathogens.

Antibody repertoire retention ( $90 \%)$ was similar in control cohorts A and B despite the longer sampling interval in B compared with A (1 year versus 3 months). The retained antibody repertoire could represent a core stable LLPC repertoire ( 90\%), and the 10\% that was lost could represent a transient repertoire derived from IgG-secreting B cells and plasmablasts. This result is consistent with previous studies that showed no effect of immunosuppressive therapy, such as B celldepleting anti-CD19 or anti-CD20 treatment, on retention of the majority of the antibody repertoire $(17,19,21,22)$. Therefore, measles is associated with greater loss of antibodymediated epitope recognition than can be explained by ablation of B cells, suggesting a direct effect on the LLPC compartment.

\section{Measles decreases the strength of epitope recognition}

Simply counting epitope numbers recognized before and after measles underestimates immune memory impairment because epitope recognition can be detected even when a large fraction of cellular clones producing the relevant antibody are eliminated. To quantify the relative abundance of particular antibodies, we generated an EBS metric, which is a z-score that measures the relative enrichment of an epitope in a VirScan immunoprecipitation (see materials and methods). Thus, EBS represents a VirScan analog of relative antibody titers and can be measured over time to detect changes in epitope recognition. Significant increases in EBS for a given epitope usually indicate a new exposure during the follow-up interval. In controls, significant reductions are normally detected when a recent exposure has occurred before the first time point, resulting in a peak around time 1 followed by an expected rapid decay of the antibody signal that is detected at time 2 .

To evaluate changes in EBS for each pathogen species in each child, EBS signals were clustered by pathogen species and child $\left(\mathrm{EBS}_{\mathrm{PC}}\right)$ and a paired $t$ test (paired per epitope) was used to test for significant differences at time 2 versus time 1. False discovery rate (fdr)adjusted $P$ values, sample size, and effect size (calculated as the fold change of the geometric mean at time 2 versus time 1) are shown for each EBS $_{\mathrm{PC}}$ cluster (Fig. 3A and fig. S4).

Significant changes in $\mathrm{EBS}_{\mathrm{PC}}$ were detected in children in each cohort. Among controls, these changes were distributed equally, below and above a fold change of 1 , indicating recent pathogen exposures before time 1 or during the follow-up interval, respectively. Unlike the controls, after measles, the significant changes in pathogen-specific $\mathrm{EBS}_{\mathrm{PC}}$ were both more frequent and nearly uniformly negative with median fold changes (0.58 and 0.62), indicating $\sim 40 \%$ reductions in EBSs (Fig. 3A). The effect was stronger after severe versus mild MV infections $(P<0.001)$. We confirmed these results by comparing the fold changes in $\mathrm{EBS}_{\mathrm{PC}}$ to quantitative ELISA assays for adenovirus, enterovirus, and respiratory syncytial virus (RSV) in a selection of children who had decreased or increased EBS, and found good correlation (correlation coefficient $r=0.73 ; P<0.001$ ) (fig. S5, A and B).

To further clarify cohort-level changes, we combined the epitopes from all individuals and evaluated changes on a per-pathogenspecies basis across each cohort (fig. S6). Among MV-infected individuals, the median fold change in EBS measured across all species was a strongly negative effect (median fold change: 0.69 or $-31 \% ; P<0.0001)$. In contrast, among controls, the antibody EBS 
was significantly changed only for relatively few pathogen species, and these were roughly balanced between positive and negative effects.

\section{Measles disrupts recognition of pathogen epitopes across the cohort}

At the cellular level, MV would not be expected to preferentially infect particular antibodysecreting cells on the basis of their pathogen targets or the type of antibodies produced (i.e., neutralizing or not). We therefore tested for cohort-wide differences in EBSs on a perepitope basis. Across approximately 1100 epitopes each from the control A and B cohorts, none significantly changed (Fig. 3, B and C, and fig. S7), indicating that antibody epitope recognition across a group of individuals is remarkably stable, even when changes are detected at the pathogen species level, as in fig. S6. In contrast, EBS was substantially reduced in $12 \%$ of the 855 epitopes evaluated after mild MV infections and in 39\% of the 1079 epitopes evaluated after severe MV infections $(P<0.0001)$ (Fig. 3, B and C). Only one epitope in mild measles and three in severe measles showed a significant increase, $<0.2 \%$ overall.

VirScan detects neutralizing antibodies when those antibodies target short contiguous epitopes encoded in the phage display. A wellcharacterized neutralizing short contiguous epitope is the 24-amino-acid target of the antiRSV monoclonal antibody therapies palivizumab and motavizumab. Among $22 \mathrm{MV}$-infected children with antibodies against this neutralizing epitope and without evidence of new RSV exposure, we detected a fold change in EBS of $0.59(\mathrm{SD} \pm 0.18 ; P<0.001)$ (fig. $\mathrm{S} 8)$, in line with the observations above and indicating that the effects of MV are the same for the neutralizing and non-neutralizing antibody repertoires.

\section{The MMR vaccine does not impair the immune repertoire}

A marked increase in the overall antibody repertoire diversity was noted in MMR-vaccinated controls (Fig. 1), indicating that a similar loss of antibodies does not appear to accompany receipt of MMR vaccines compared with MV infections. However, in infants and young children, such as the MMR-vaccinated cohort in this study, the antibody repertoire continues to add antibody diversity over time (fig. S9). This is particularly true during the second year of life, following depletion of maternal antibodies, when measles vaccines are first given. These overall increases in antibody diversity over time could be obscuring potential minor impairments from measles vaccine, especially given the relatively long sampling interval for the vaccinated controls. To determine whether viral responses before or after
MMR vaccination might be impaired, we analyzed EBS for antibodies detected surrounding MMR vaccine. We found no overall change in EBS after MMR vaccination (fig. S10), which is consistent with observations that the MV vaccine strains are not associated with widespread infection of CD150 $0^{+}$lymphocytes $(27,28)$.
Thus, this higher-resolution analysis failed to detect immune impairment at the epitope level. Combined with increased epitope diversity after MMR vaccination (Fig. 1A), this analysis supports decades of observations that MMR vaccines do not increase susceptibility to subsequent infections $(29,30)$.

A One point $=$ all epitopes from one pathogen per child

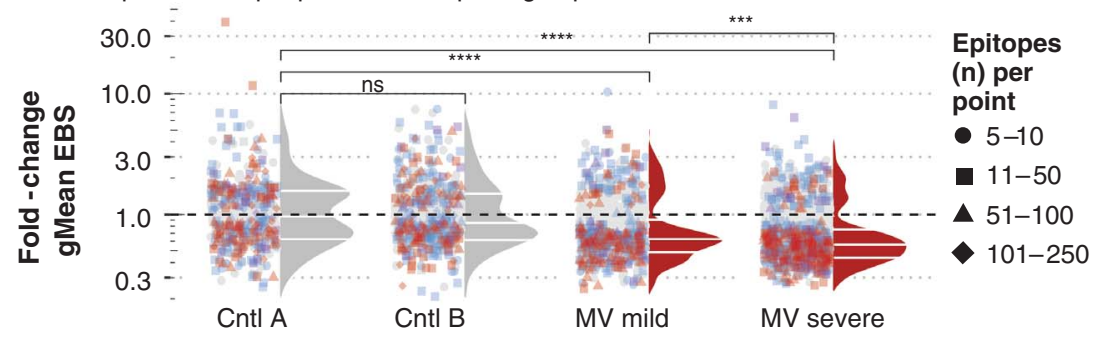

B One point $=$ one epitope across children

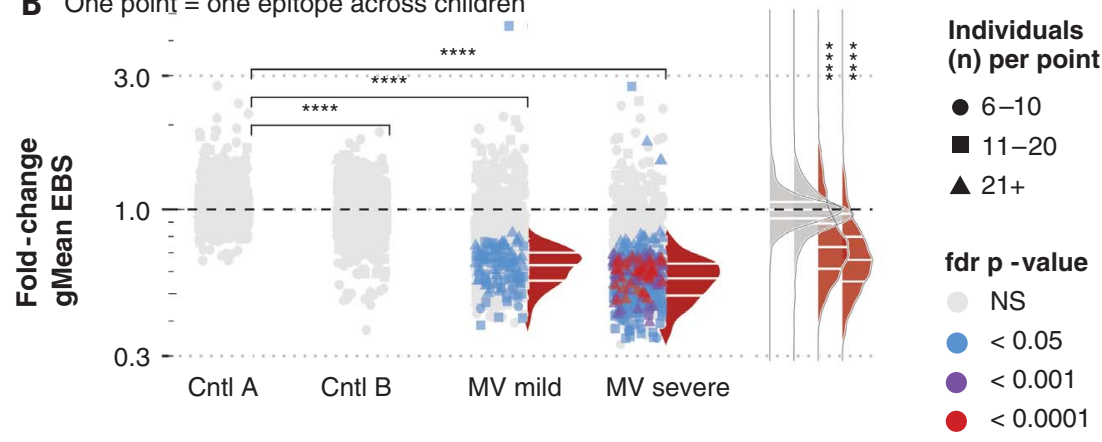

C One point $=$ all epitopes across all children

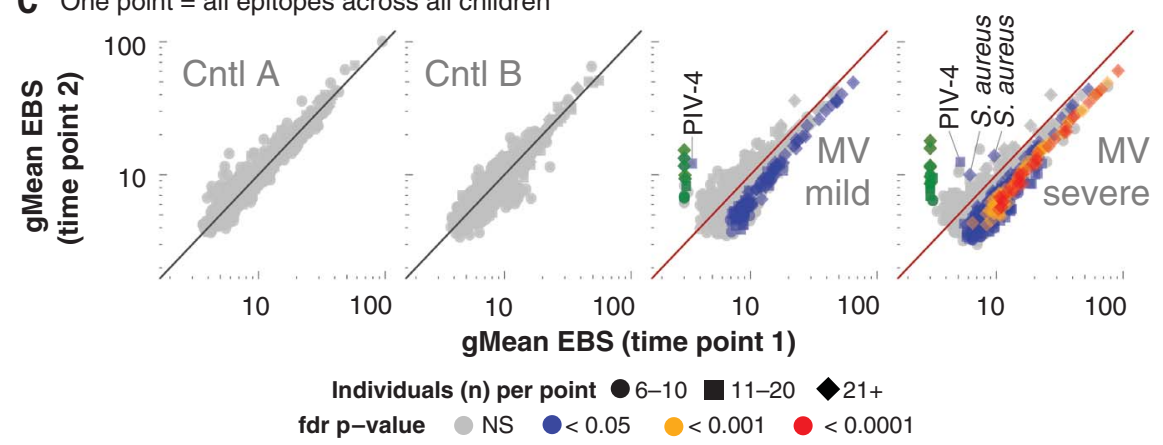

Fig. 3. Measles virus infections are associated with diminished epitope binding signal. Epitope binding signal (EBS) was measured for each epitope. (A) Each point represents one pathogen species per sample pair. Changes in EBS for all epitopes recognized per pathogen per sample pair were compared using a Wilcoxon matched-pairs signed rank test. Fdr-adjusted $P$ values (adjusted for a fdr of $5 \%$ ) are indicated by color of each symbol. The total number of epitopes included in each paired test (i.e., the number of epitopes recognized for each species per paired sample) are indicated by the symbol shape. For each pathogen, the geometric mean (gMean) EBS was calculated at each time and the fold change at time 2 versus time 1 was calculated and indicated by the position of each point along the $y$ axis. Density distributions adjacent to the points reflect the distribution of points that were significantly changed on the basis of a $\mathrm{fdr} P$ value $<0.05$. Only pathogens with antibodies targeting $\geq 5$ epitopes were included. (B) Change in gMean EBS per epitope, across all paired samples per cohort. Format as in (A), except here each point represents a single epitope, and the gMean EBS is calculated across all paired samples with the epitope detected. The color of each point represents the fdr-adjusted Wilcoxon matched-pairs signed rank test $P$ value, and shapes indicate the number of paired samples per cohort with antibodies against the particular epitope). Only epitopes recognized in $>6$ paired samples were included. (C) Scatter plot showing each epitope (point) shown in (B), but instead of plotting the fold change, the gMean EBS at time 1 ( $x$ axis) versus time 2 ( $y$ axis) is plotted. The $P$ values (colors) and samples sizes (shapes) are indicated. MV-specific epitopes were included and highlighted in green. Epitopes significantly changed in a positive direction are labeled with pathogen species name. NS, not significant; PIV-4, parainfluenza virus-4. 
Nevertheless, because of the naturally rapidly increasing antibody diversity in this young cohort at the time of MMR receipt, we cannot definitively rule out the potential for minor reductions of antibody-producing cells with measles vaccination.

\section{Reconstruction of the antibody repertoire with high-transmission pathogens is spatially clustered}

Although antibody diversity and abundance were negatively affected for almost all pathogens, a subset of children had increased EBS and/or epitope hits for particular pathogens after measles (Figs. 1, D and E, and 3A; and figs. S4, S8, and S11), suggestive of potential restoration of immune memory after the initial immune depletion.

Across all of the pathogen-child combinations with significantly increased EBS after measles, $80 \%$ were attributable to only six pathogens: adenovirus $\mathrm{C}$, influenza A virus, respiratory syncytial virus, human herpesvirus 4 (Epstein-Barr virus; HHV-4), Streptococcus pneumoniae and Staphylococcus aureus. In these children, the probability of retaining preexisting antibodies for these pathogens was relatively high (fig. S12).

If reconstruction of the antibody repertoire requires new exposures, children experiencing repertoire reconstruction for transmissible pathogens should cluster spatially. Using postal codes and household identifiers, we observed clustering of pathogen-specific repertoire reconstruction at both the postal code or school level and the household level (Fig. 4). Among 19 children with increased adenovirus $\mathrm{C}$ antibody EBS, eight (42\%) came from a single postal code (number 7) that included only 12 (16\%) of the 77 measles cases (Fig. 4A). Thus, children in this postal code had significantly increased odds [odds ratio (OR) 9.4; Fisher's exact test, $P<0.001]$ of recovering their adenovirus $\mathrm{C}$ repertoire versus other postal codes, suggesting recovery is associated with pathogen transmission. Moreover, six of these children $(75 \%)$ shared a household with another child with increased adenovirus C EBS. We found similar clustering effects for other respiratory pathogens: influenza A virus [of eight children with increased influenza A EBS, five (63\%) were from a single postal code representing only $26 \%$ of the measles cases (OR 5.8; $P<0.05)$ and two were from the same household; Fig. 4B]; RSV [of nine with increased EBS, five (56\%) were from the same postal code (OR 4.5; $P<0.05$ ) and four of them (44\%) shared a house; Fig. 4C]; rhinovirus [of 12 with increased EBS, eight (67\%) shared a household with at least one other child with increased rhinovirus EBS]; and S. pneumoniae [of 13 , seven (54\%) came from a single postal code (OR 4.5; $P<0.05$ ) and two shared a household]. Combined, these indicate local pathogen transmission and suggest that reconstruction of the antibody repertoire occurs on a per-pathogen basis and is associated with new exposures.

In contrast to the highly transmissible respiratory pathogens above, where mean EBS was increased after measles infection, we found that for chronic viruses [i.e., HHV-4 and human herpesvirus 5 (cytomegalovirus; HHV-5)], there was no evidence of spatial clustering. Further, only 6 out of 12 individuals (50\%) with increased HHV-4 antibody EBS also developed antibodies against new HHV-4 epitopes. HHV-5 behaved similarly, with only $33 \%$ of individuals with increased HHV-5 antibody EBS also developing new antibodies to previously untargeted HHV-5 epitopes. The lack of development of antibodies targeting new epitopes, despite increases in EBS of existing antibodies for these two pathogens, is different from what we observed for the moretransmissible viruses noted above, in which $90 \%$ of pathogen-specific increases in EBS at time 2 were associated with development of new antibodies against previously unrecognized pathogen-specific epitopes. An increase in EBS without addition of new epitope recognition points toward reinfection with or reactivation of the same virus.

Two additional notable pathogens for which positive changes in EBS were often detected after measles were the bacteria S. pneumoniae
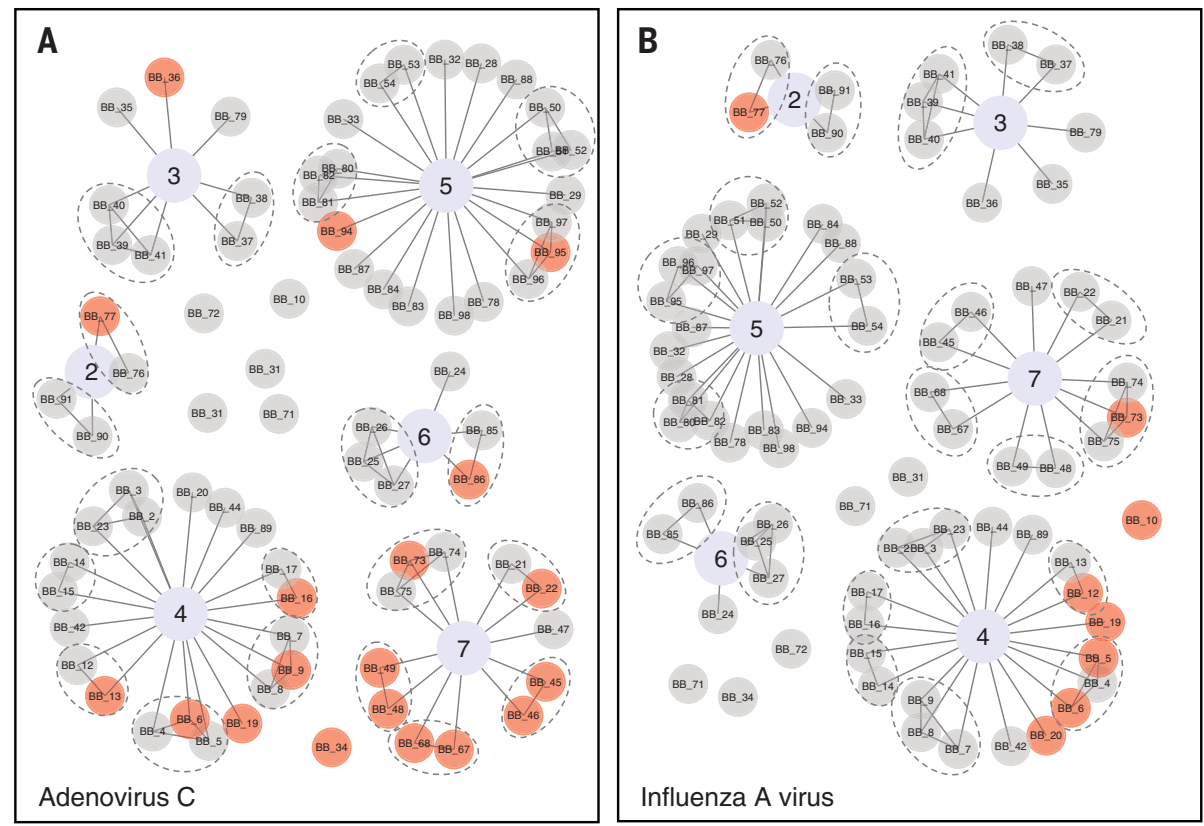

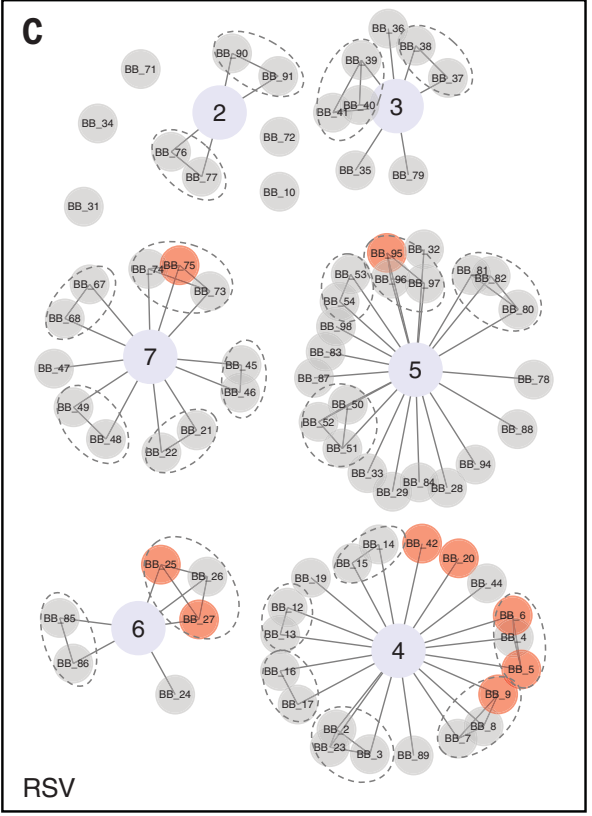

Fig. 4. Increases in antibody epitope binding signal cluster within households and in postal units. We tested whether individuals with increased EBS indicate new exposures due to pathogen transmission by looking for evidence of spatial clustering among children with increases in EBS. In (A to $\mathbf{C}$ ), each child (node) is connected by an edge to their postal code (indicated by a central node with an assigned value, 2 through 7). Children shown as single nodes were the only children studied from their postal code. Children from the same household are connected by an edge and encircled by dashed gray ovals. Each of the three viruses where increases in EBS were most common are shown: (A) adenovirus C, (B) influenza A virus, and $(C)$ respiratory syncytial virus. Children with significantly increased EBS for the pathogen (indicating exposure during the interval) are highlighted with red circles and those with decreased or unchanged EBS with gray circles. 
and $S$. aureus. Acute viral respiratory infections, including measles, increase susceptibility to respiratory bacterial infections $(6,31-33)$. After measles, new acquisitions of bacteria or increased replication of already colonizing bacteria could elicit antibody responses and reinstate antibody diversity and protection against these pathogens. In line with this, we observed net increases in antibody diversity, including development of antibodies against new epitopes, and increases in EBS for $S$. aureus [the only pathogen for which a net positive change in antibody diversity or EBS was detected after measles; 45 of 77 children (58\%) had increased EBS] (Fig. 1 and fig. S11). Increases were also observed for S. pneumoniae, although the overall cohort-level effect remained negative.

\section{Reconstruction of the antibody repertoire through reexposure after measles carries risks}

Despite the potential benefits of reinstating the antibody repertoire, exposure to pathogens after measles-especially when in the presence of diminished preexisting immune memory- can carry risks. Ten of 43 children (23\%) with severe measles were diagnosed with acute otitis media (AOM; most commonly pneumococcal), which was associated with a threefold increase in odds of carrying greater pneumococcal antibody diversity after measles compared with children without an AOM diagnosis. In addition, two children with mild measles and AOM and one with a diagnosis of bacterial pneumonia had increased pneumococcal antibody diversity at follow-up. Combined, these effects suggest that the antibody repertoire begins rebuilding soon after measles, through pathogen exposures, and that pathogen exposure after measles, while serving to reinstate immune memory, may pose excess risk.

\section{Experimental MV infection confirms a decrease in previously acquired immune memory}

To confirm the findings above using a controlled experimental setting with longer followup, we used VirScan to profile the antibody repertoire in plasma collected before and 5 months after experimental MV infection of four rhesus macaques (34). The overall diversity of the antibody repertoire was decreased an average of $26 \%$ (range: 21 to $35 \%$ ), and reductions were distributed across pathogens (Fig. 5A). Notable exceptions were observed for simian foamy virus and Epstein-Barr virus, both of which can reactivate during immune dysregulation and thus may be early contributors to repertoire reconstruction. As expected, we detected increases in recognition of MV epitopes (Fig. 5B). Out of $21 \mathrm{MV}$ epitopes recognized after measles, only one (phosphoprotein/V protein 29) was recognized before infection and was reduced by $40 \%$. This likely represented a cross-reactive epitope that was diminished along with the rest of the repertoire.

Each monkey lost, on average, 40 to $60 \%$ of its preexisting antibody repertoire (Fig. 5C), and this loss persisted for at least 5 months after MV infection. In each monkey, the fractions lost were distributed across the pathogens, which is expected for a virus that indiscriminately infects antibody-secreting cells.
Fig. 5. Measles virus infection in macaques deletes preexisting immune memory. Four rhesus macaques (14Y, 31Y, 46Y, and 50Y) were infected with the Bilthoven strain of wild-type MV [detailed in (34)], and plasma samples were collected before and 5.1 months after infection. (A) Heatmap showing the percentage change in total epitopes recognized per pathogen in each monkey from time 1 to time 2. Pathogens with $>10$ unique epitopes recognized at either time point per macaque are shown.

The color and text indicate percent change in total epitopes recognized per pathogen species before versus after infection. (B) Heatmap showing the signal strength of anti-measles antibodies (EBS) for each epitope for which at least one monkey developed an antibody. Colors and text of each cell represent the respective EBS values.Letters indicate the MV protein ( $F$, fusion protein; $\mathrm{H}$, hemagglutinin; $\mathrm{N}$, nucleoprotein; and P, phosphoprotein), and the numbers indicate the position along the protein of the first amino acid of the 56-amino-acid peptide. (C) The fractions of epitopes recognized before MV infection that remained 5 months after are shown (0 months after infection is baseline and thus set to 1 for all pathogens). Each gray line indicates a different pathogen, and the dark black line indicates the average across the pathogens. The boxplot summarizes the interquartile range and median fraction retained across the pathogens.
B

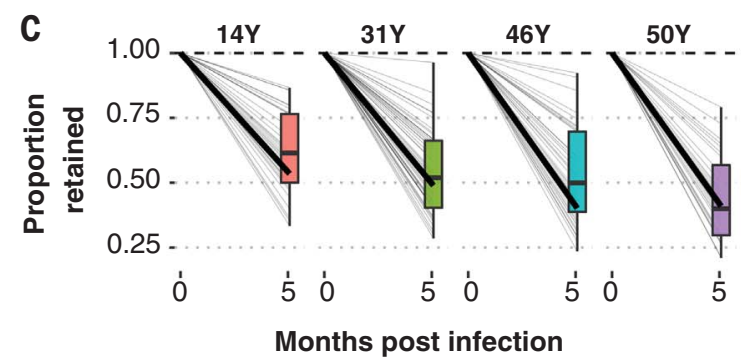

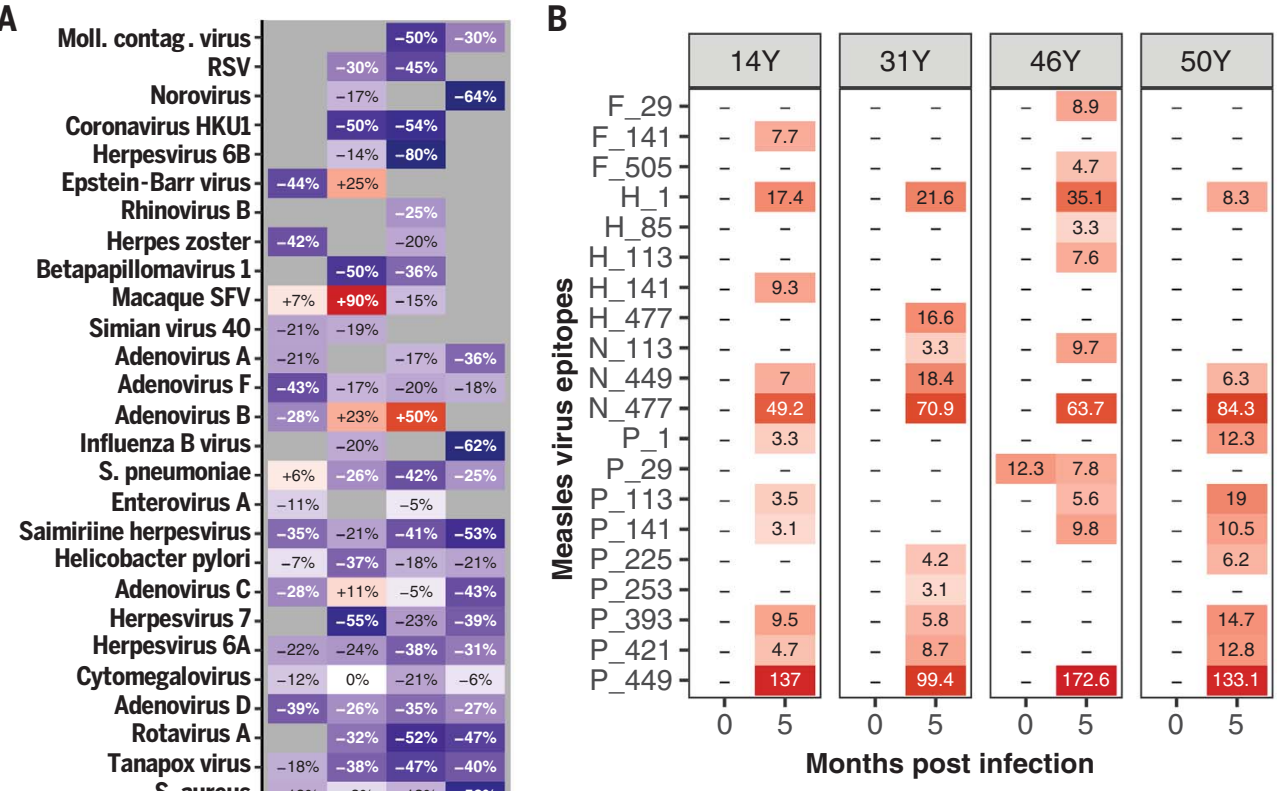




\section{Cumulative evidence supports the establishment of an immune amnesia state after measles}

Using VirScan, we quantified the effects of measles on antipathogen antibody repertoires in plasma obtained before and after natural and experimental MV infections. We found that measles is associated with large reductions in both the diversity of the antibody repertoire and magnitude of the binding signal, likely reflecting reduced antibody titers resulting from diminished numbers of cells producing the respective antibody. The reduction of diversity may be greater than we report because even if particular antibody-producing cells have been eliminated, an antibody halflife of $\sim 3$ weeks (35) means that residual antibodies were still detectable at the time of sampling. Our findings show that after recovery from MV infections, individuals enter a state in which immune functionality is restored, but memory cell elimination induced by measles may alter previously acquired memory.

MV can infect 20 to $70 \%$ of memory cells, including B cells, T cells, and plasma cells in the lymphoid tissue and peripheral blood during the first 3 to 10 days after infection $(7,10,24)$. Clinical trials of systemic administration of engineered MV for oncolytic therapy of multiple myeloma also demonstrated efficient entry of MV into the bone marrow and plasma cell infection (36). In wild-type MV infections, lymphocytes undergo rapid proliferation during immune activation (37), and lymphocyte counts (and other immunological markers) return to normal levels within weeks of infection. Thus, the immunological toll of measles is often considered limited to that period. After experimental MV infection in monkeys, we found that we could no longer detect up to $60 \%$ of the antibody repertoire, and this persisted for at least 5 months. This loss could be permanent, owing to elimination of B cells as well as LLPCs (11), although we cannot rule out possible involvement of additional unknown pleiotropic effects that may also cause antibody-secreting cells to down-regulate antibody production. Furthermore, because T cells also express SLAM and are infected by MV, T cell immunity may be diminished through similar mechanisms, and this could explain the loss of cutaneous tuberculin reactions after measles (5). Lasting remissions of autoimmune-related disorders after measles have been detected by researchers since the 1940s (38), supporting the potential persistence of immune suppression and pointing to persistent long-term effects. Because LLPCs are terminally differentiated and do not replicate (39), the rebuilding of immune memory after measles-induced LLPC elimination would likely require reexposures, either through natural infection or vaccination; thus it could potentially take months or years to return the immune repertoire back to baseline for all relevant pathogens. During the rebuilding process, individuals would potentially be at increased risk for other infectious diseases. Revaccination with routine childhood vaccines after measles could help to mitigate long-term suffering that might stem from immune amnesia and subsequent increased susceptibility to other infections.

We previously reported evidence that measles epidemics link to population mortality 2 to 3 years later $(15,40)$. We hypothesized that the observed dynamics could potentially be explained by an immunomodulatory effect of measles, similar to what we show here. We found no such debilitative effects for the live MMR vaccine (15). Furthermore, because, in the prevaccine era, MV infected nearly all children within the first decade of life, the vaccine may have contributed to considerably greater benefits by preventing measles and immune amnesia. By preserving immunity, measles vaccines may have reset overall baseline morbidity and mortality rates to lower levels (15).

Given the variation in the degree of immune repertoire modulation we observed, we anticipate that future risk of morbidity and mortality after measles would not be homogeneous but would be skewed toward individuals with the most severe elimination of immunological memory. These findings underscore the crucial need for continued widespread vaccination. More than 7 million people are estimated to have been infected with measles in 2018 (1-3). Comprehensive coverage with MV vaccine would not only help prevent the $>120,000$ deaths that will be directly attributed to measles this year, but, by preventing MV immune amnesia and thus preserving immunity, MV vaccines could also avert potentially hundreds of thousands of additional deaths attributable to the lasting damage to the immune system. The WHO recently reported that between 2000 and 2017, MV vaccines have prevented more than 21 million deaths directly attributable to measles (41). These findings suggest that the number of deaths averted might be much greater, and they attest to the immense public health value of the measles vaccine.

\section{REFERENCES AND NOTES}

1. A. Dabbagh et al., MMWR Morb. Mortal. Wkly. Rep. 67 1323-1329 (2018).

2. World Health Organization, New measles surveillance data for 2019 (2019); www.who.int/immunization/newsroom/measlesdata-2019/en/

3. A. Portnoy et al., Lancet Glob. Health 7, e472-e481 (2019).

4. H. S. Goodridge et al., Nat. Rev. Immunol. 16, 392-400 (2016).

5. C. Pirquet, Dtsch. Med. Wochenschr. $34,1297-1300$ (1908).

6. D. E. Griffin, Immunol. Rev. 236, 176-189 (2010).

7. R. L. de Swart et al., PLOS Pathog. 3, e178 (2007).
8. H. Tatsuo, N. Ono, K. Tanaka, Y. Yanagi, Nature 406, 893-897 (2000).

9. B. Hahm, N. Arbour, M. B. Oldstone, Virology 323, 292-302 (2004).

10. R. D. de Vries et al., PLOS Pathog. 8, e1002885 (2012).

11. J. De Salort, J. Sintes, L. Llinàs, J. Matesanz-Isabel, P. Engel, Immunol. Lett. 134, 129-136 (2011).

12. B. M. Laksono et al., J. Virol. 92, e00131-18 (2018).

13. J. J. Ryon, W. J. Moss, M. Monze, D. E. Griffin, Clin. Diagn. Lab. Immunol. 9, 994-1003 (2002).

14. V. G. Tamashiro, H. H. Perez, D. E. Griffin, Pediatr. Infect. Dis. J. 6, 451-453 (1987)

15. M. J. Mina, C. J. E. Metcalf, R. L. de Swart, A. D. M. E. Osterhaus, B. T. Grenfell, Science 348, 694-699 (2015).

16. R. D. de Vries, R. L. de Swart, PLOS Pathog. 10, e1004482 (2014).

17. P. Isnard et al., Proc. Natl. Acad. Sci. U.S.A. 116, 10899-10904 (2019).

18. J. L. Halliley et al., Immunity 43, 132-145 (2015).

19. E. Hammarlund et al., Nat. Commun. 8, 1781 (2017).

20. I. J. Amanna, N. E. Carlson, M. K. Slifka, N. Engl. J. Med. 357, 1903-1915 (2007)

21. M. D. Pescovitz et al., J. Allergy Clin. Immunol. 128, 1295-1302.e5 (2011).

22. J. Hill et al., Blood Adv. 10.1182/bloodadvances.2019000717 (2019)

23. K. Gadroen et al., BMJ Open 8, e021465 (2018).

24. B. M. Laksono et al., Nat. Commun. 9, 4944 (2018).

25. G. J. Xu et al., Science 348, aaa0698 (2015)

26. H. B. Larman et al., Nat. Biotechnol. 29, 535-541 (2011).

27. R. S. van Binnendijk, R. W. van der Heijden, G. van Amerongen, F. G. UytdeHaag, A. D. Osterhaus, J. Infect. Dis. 170, 443-448 (1994)

28. R. L. de Swart et al., NPJ Vaccines 2, 22 (2017)

29. M. M. Coughlin, A. S. Beck, B. Bankamp, P. A. Rota, Viruses 9 11 (2017)

30. W. J. Moss, D. E. Griffin, Nat. Rev. Microbiol. 4, 900-908 (2006).

31. M. J. Mina, J. A. McCullers, K. P. Klugman, mBio 5, e01040-13 (2014).

32. M. J. Mina, K. P. Klugman, Lancet Respir. Med. 2, 750-763 (2014).

33. R. Cole, J. Am. Med. Assoc. 70, 1147-1153 (1918)

34. A. N. Nelson et al., Sci. Rep. 7, 11474 (2017).

35. P. R. Hinton et al., J. Immunol. 176, 346-356 (2006).

36. A. Dispenzieri et al., Leukemia 31, 2791-2798 (2017).

37. D. E. Griffin, B. J. Ward, E. Jauregui, R. T. Johnson, A. Vaisberg, N. Engl. J. Med. 320, 1667-1672 (1989).

38. R. W. Blumberg, H. A. Cassady, Am. J. Dis. Child. 73, 151-166 (1947)

39. M. K. Slifka, R. Antia, J. K. Whitmire, R. Ahmed, Immunity 8 , 363-372 (1998)

40. M. J. Mina, B. T. Grenfell, C. J. E. Metcalf, Science 365 eaax6498 (2019)

41. World Health Organization, Measles fact sheet (2019); www.who.int/news-room/fact-sheets/detail/measles.

42. M. J. Mina, S. J. Elledge, Measles Immune Amnesia: Data and Code for Mina et al. Science 2019, Dryad (2019): https://doi.org/10.5061/dryad.5tb2rbp0j.

\section{ACKNOWLEDGMENTS}

We express our gratitude to the participants, including children parents, school staff, and other volunteers, who provided time and samples for this work. We also thank B. T. Grenfell and C. J. E. Metcalf for their early insights into this work and E. Shrock for assistance with assays. Funding: This work was supported by a grant from the Value of Vaccine Research Network to S.J.E. and M.J.M.; a grant from the Gates Foundation to S.J.E.; an NIH/NIAID U24 grant to H.B.L. and S.J.E.; a grant from the European Union Seventh Framework Program (grant 202063) and a grant from the Academy of Finland (Centre of Excellence in Molecular Systems Immunology and Physiology; grant 250114) to M.K.; NIH R01 DK032493 to M.R.; NIH R21 Al095981 and R01 Al131228 to D.E.G.; and a grant from PREPARE Europe (EU FP7 grant 602525) to R.L.d.S. S.J.E. is an Investigator with the Howard Hughes Medical Institute. Author contributions: Conceptualization: M.J.M., T.K., R.L.d.S., and S.J.E.; Investigation: M.J.M., T.K., Y.L., and M.L.; Reagents and samples: H.S., M.K., D.F.C., M.R., M.S.W., D.E.G., A.N.N., H.B.L., R.D.d.V., and R.L.d.S.; Writing: M.J.M., T.K., and S.J.E.; Supervision: S.J.E. Competing interests: M.S.W. and D.F.C. are employees of Genentech. S.J.E. is a founder of TSCAN Therapeutics, MAZE Therapeutics, and Mirimus. S.J.E. serves on the scientific advisory boards of CRISPR Therapeutics, Homology Medicines, TSCAN Therapeutics, and XChem and is 
an adviser for MPM Capital, none of which affect this work. M.J.M. has served as a member of Sanofi advisory board for RSV therapeutics. D.E.G. is a member of the GlaxoSmithKline

Vaccine Research and Development Advisory Board. S.J.E., T.K. and H.B.L. are inventors on a patent application filed by The Brigham and Women's Hospital (US20160320406A) that covers the use of the VirScan library to identify pathogen antibodies in blood. All other authors declare no competing interests. Data and materials availability: The VirScan library is available under a material transfer agreement. Data are available in the main text, the supplementary materials, or on Dryad (42)

\section{SUPPLEMENTARY MATERIALS}

science.sciencemag.org/content/366/6465/599/suppl/DC1 Materials and Methods
Figs. S1 to S12

Table S1

References (43-45)

View/request a protocol for this paper from Bio-protocol.

9 July 2019; accepted 30 September 2019

10.1126/science.aay6485 


\section{Science}

\section{Measles virus infection diminishes preexisting antibodies that offer protection from other pathogens}

Michael J. Mina, Tomasz Kula, Yumei Leng, Mamie Li, Rory D. de Vries, Mikael Knip, Heli Siljander, Marian Rewers, David F. Choy, Mark S. Wilson, H. Benjamin Larman, Ashley N. Nelson, Diane E. Griffin, Rik L. de Swart and Stephen J. Elledge

\section{The toll of measles on the immune system}

Many of the deaths attributable to measles virus are caused by secondary infections because the virus infects and functionally impairs immune cells. Whether measles infection causes long-term damage to immune memory has been unclear. This question has become increasingly important given the resurgence in measles epidemics worldwide. Using a blood test called VirScan, Mina et al. comprehensively analyzed the antibody repertoire in children before and after natural infection with measles virus as well as in children before and after measles vaccination. They found that measles infection can greatly diminish previously acquired immune memory, potentially leaving individuals at risk for infection by other pathogens. These adverse effects on the immune system were not seen in vaccinated children. Science, this issue p. 599

ARTICLE TOOLS

SUPPLEMENTARY MATERIALS

RELATED CONTENT

REFERENCES

PERMISSIONS http://science.sciencemag.org/content/366/6465/599

http://science.sciencemag.org/content/suppl/2019/10/30/366.6465.599.DC1

http://science.sciencemag.org/content/sci/366/6465/560.full http://immunology.sciencemag.org/content/immunology/4/41/eaaz4195.full http://stm.sciencemag.org/content/scitransmed/10/433/eaao5945.full http://stm.sciencemag.org/content/scitransmed/6/232/232ra52.full http://immunology.sciencemag.org/content/immunology/4/41/eaay6125.full

This article cites 41 articles, 9 of which you can access for free http://science.sciencemag.org/content/366/6465/599\#BIBL

http://www.sciencemag.org/help/reprints-and-permissions

Use of this article is subject to the Terms of Service

Science (print ISSN 0036-8075; online ISSN 1095-9203) is published by the American Association for the Advancement of Science, 1200 New York Avenue NW, Washington, DC 20005. The title Science is a registered trademark of AAAS.

Copyright $@ 2019$ The Authors, some rights reserved; exclusive licensee American Association for the Advancement of Science. No claim to original U.S. Government Works 